\title{
Molecular Characterization of HOXA13 Polyalanine Expansion Proteins in Hand-Foot-Genital Syndrome
}

\author{
Boris Utsch, ${ }^{1}$ Colleen D. McCabe, ${ }^{2}$ Kenneth Galbraith, ${ }^{2}$ Ricardo Gonzalez, ${ }^{3}$ Mark Born, ${ }^{4}$ \\ Jörg Dötsch, ${ }^{1}$ Michael Ludwig, ${ }^{5}$ Heiko Reutter, ${ }^{6}$ and Jeffrey W. Innis ${ }^{2 *}$ \\ ${ }^{1}$ Children Hospital, University of Erlangen-Nuremberg, Erlangen, Germany \\ ${ }^{2}$ Departments of Human Genetics and Pediatrics, University of Michigan, Ann Arbor, Michigan \\ ${ }^{3}$ A.I. duPont Hospital for Children, Wilmington, Delaware \\ ${ }^{4}$ Department of Radiology, Section of Pediatric Radiology, University of Bonn, Bonn, Germany \\ ${ }^{5}$ University of Bonn, Bonn, Germany \\ ${ }^{6}$ Department of Human Genetics, University of Bonn, Bonn, Germany
}

Received 13 April 2007; Accepted 8 June 2007

\begin{abstract}
We report on a father and daughter with hand-foot-genital syndrome (HFGS) with typical skeletal and genitourinary anomalies due to a 14-residue polyalanine expansion in HOXA13. This is the largest (32 residues) polyalanine tract so far described for any polyalanine mutant protein. Polyalanine expansion results in protein misfolding, cytoplasmic aggregation and degradation; however, HOXA13 polyalanine expansions appear to act as loss of function mutations in contrast to gain of function for HOXD13 polyalanine expansions. To address this paradox we examined the cellular consequences of polyalanine expansions on HOXA13 protein using COS cell transfection and immunocytochemistry. HOXA13 polyalanine expansion proteins form cytoplasmic aggregates, and distribution between cytoplasmic aggregates or the nucleus is polyalanine tract
\end{abstract}

size-dependent. Geldanamycin, an Hsp90 inhibitor, reduces the steady-state abundance of all polyalanine-expanded proteins in transfected cells. We also found that wild-type HOXA13 or HOXD13 proteins are sequestered in HOXA13 polyalanine expansion cytoplasmic aggregates. Thus, the difference between HOXA13 polyalanine expansion loss-offunction and HOXD13 polyalanine expansion dominantnegative effect is not the ability to aggregate wild-type group 13 paralogs but perhaps to variation in activities associated with refolding, aggregation or degradation of the proteins. (c) 2007 Wiley-Liss, Inc.

Key words: Hand-foot-genital syndrome; HOXA13; polyalanine expansion

How to cite this article: Utsch B, McCabe CD, Galbraith K, Gonzalez R, Born M, Dötsch J, Ludwig M, Reutter H, Innis JW. 2007. Molecular characterization of HOXA13 polyalanine expansion proteins in hand-foot-genital Syndrome. Am J Med Genet Part A 143A:3161-3168.

\section{INTRODUCTION}

Trinucleotide repeat expansions in human genetic diseases may occur in noncoding sequences, transcribed but not translated sequences, or within translated sequences for polyglutamine and polyalanine tracts [Albrecht and Mundlos, 2005]. In-frame polyalanine expansions encoded by imperfect triplet repeats have been reported in several diseases. The expansion mutations are meiotically and mitotically stable and exert their effects through different mechanisms [Kjaer et al., 2002, 2005; Lavoie et al., 2003; Brown and Brown, 2004].

Hand-foot-genital syndrome (HFGS) is a rare autosomal dominant disorder caused by mutations in the HOXA13 gene [Mortlock and Innis, 1997]. It presents with a highly penetrant distal limb pattern including short first metacarpals, small distal phalanges of the thumbs, short middle phalanges of the fifth fingers, and fusion or delayed ossification of carpals/tarsals. The great toe is shorter due to a short first metatarsal and a small, pointed distal phalanx. Genitourinary tract anomalies are incompletely penetrant and highly variable in severity [reviewed in Innis, 2004].

This article contains supplementary material, which may be viewed at the American Journal of Medical Genetics website at http://www. interscience.wiley.com/jpages/1552-4825/suppmat/index.html.

Boris Utsch and Colleen D. McCabe contributed equally to this work. Grant sponsor: NIH; Grant number: HD37486.

*Correspondence to: Jeffrey W. Innis, Department of Human Genetics, 4811 Med Sciences II, Box 0618, 1500 E. Medical Center Drive, Ann Arbor, MI 48109-0618. E-mail: innis@umich.edu

DOI 10.1002/ajmg.a.31967 
Various HOXA13 mutations have been described in HFGS including missense, nonsense, and polyalanine tract expansions. HOXA13 polyalanine expansion mutations appear to lead to loss of function. There are three, large homopolymeric polyalanine tracts of lengths 14,12 , and 18 residues encoded within wild-type exon 1 of HOXA13. Polyalanine expansions in all three large alanine tracts have been associated with HFGS, and in a mouse model of HOXA13 polyalanine expansion of the third largest repeat, the mutant mRNA is stable yet the protein is degraded [Innis et al., 2004]. In contrast, HOXD13 polyalanine expansion mutations act in a dominant negative manner with increasing severity associated with increases in polyalanine tract length. Recently, Albrecht et al. [2004] showed that numerous polyalanine-expanded transcription factors, including mutant HOXA13 or HOXD13 are misfolded and become localized in cytoplasmic aggregates. Importantly, wild-type HOXD13 protein also was sequestered within HOXD13 polyalanine cytoplasmic aggregates in cotransfection experiments in COS cells, whereas, wild-type, cotransfected HOXD11 or HOXD12 were not. They also showed that geldanamycin treatment of HOXD13 polyalanine transfected cells resulted in a reduction in cytoplasmic aggregation and greater nuclear localization of the mutant protein.

In this report, we describe a newly recognized family with HFGS carrying the largest HOXA13 polyalanine expansion ( +14 alanines) observed thus far. The skeletal phenotype in this family is not different from other HFGS individuals, however the proband, but not her affected father, had tethered cord and talipes equinovarus, findings previously unreported in any patient with HFGS. To explore the nature of the difference in HOXA13 polyalanine (null) versus HOXD13 polyalanine (dominant-negative) expansion effects, we studied HOXA13 +10 and +14 polyalanine mutant proteins using COS cell transfection and immunocytochemistry.

\section{MATERIALS AND METHODS}

\section{Probands and Family}

Genomic DNA was extracted from blood leukocytes of the family members after informed consent was obtained. The research project was approved by the Ethics Committee of the University of Bonn (No. 006/04).

PCR analysis of the coding sequence of HOXA13 was accomplished according to prior protocols [Innis et al., 2004], and sequencing of the both strands of the corresponding PCR products was performed. Furthermore, separate alleles were cloned into the pGEM T-easy Promega expression vector. Results presented were on at least two independent PCR amplifications followed by DNA sequencing of gelpurified PCR products.

\section{Plasmids}

To create pCMV-HOXA13 $3^{\text {ala28 }}$ (referred to as +10 HOXA13), wild-type murine HOXA13 cDNA was cleaved with SfiI removing a 216 bp fragment leaving orientation specific cohesive ends and replaced with a double-stranded oligonucleotide synthesized to this region with an additional 30 nucleotides coding 10 alanine residues described previously [Innis et al., 2004]. The +10 HOXA13 cDNA was then cleaved with BglII and XbaI resulting in a 1,496 bp fragment, which was cloned into plasmid pCMV5.

The construction of the +14 HOXA13 mutant template required insertion of the mutated human segment as a replacement for the homologous sequence in a pre-constructed mouse Hoxa13 cDNA template. To create pCMV-HOXA13 $3^{\text {ala } 32}$ (referred to as +14 HOXA13), patient DNA was amplified with primers designed to nucleotides 76-109 bp and 512$532 \mathrm{bp}$ from the human HOXA13 cDNA. The $498 \mathrm{bp}$, expanded allele was purified away from the $456 \mathrm{bp}$, wild-type allele on a $2 \%$ agarose gel (data not shown). SacI digestion of the expanded allele resulted in a $443 \mathrm{bp}$ fragment that was cloned into SacI digested wild-type murine HOXA13 cDNA replacing a $401 \mathrm{bp}$ murine wild-type fragment. The +14 HOXA13 cDNA was cleaved with BglII and XbaI resulting in a 1,508 bp fragment, which was cloned into pCMV5. A mouse +14 HOXD13 polyalanine expansion expression plasmid was generously provided by Stefan Mundlos, Berlin, Germany.

\section{Cell Lines and Transfections}

COS-7 cells were grown in high glucose DMEM media (Invitrogen, Carlsbad, CA) supplemented with $10 \%$ FBS, $100 \mathrm{U} / \mathrm{ml}$ penicillin, $100 \mu \mathrm{g} / \mathrm{ml}$ streptomycin, and $2 \mathrm{mM}$ L-glutamine (GIBCO-BRL). COS7 $\left(1.25 \times 10^{5}\right)$ cells were plated in $2 \mathrm{ml}$ complete media per well in six-well plates and allowed to adhere overnight. Transfections were carried out using Fugene-6 (Roche Applied Sciences, Indianapolis, IN) according to the manufacturer's suggestions.

\section{Immunocytochemistry}

Cells were grown on Growth Coverslips (Fisher, Pittsburgh, PA) and were fixed $24 \mathrm{hr}$ after transfection with $4 \%$ paraformaldehyde for $5 \mathrm{~min}$ are room temperature. Cells were permeabilized with $0.5 \%$ Triton in PBS for 5 min are room temperature. Fixed cells were incubated with 1:200 rabbit antiHOXA13 [Post et al., 2000] or 1:200 rabbit antiHOXD13 [McCabe and Innis, 2005] in PBS with normal goat serum (Vector, Burlingame, CA) for $1 \mathrm{hr}$ at room temperature, washed in PBS, and incubated with 1:500 anti-rabbit-Rhodamine (Santa Cruz Biotechnology, Santa Cruz, CA) and 1:100 anti-FLAG M2-FITC (Sigma, St. Louis, MO), when appropriate, 
diluted in PBS with 1\% normal goat serum for $1 \mathrm{hr}$ at room temperature. Coverslips were then mounted with VECTASHIELD containing DAPI (Vector Labs) and images were collected using a Zeiss Axiophot microscope $(100 \times$ objective).

\section{Western Blotting}

COS7 cells, which exhibit no endogenous expression of HOXA13 or HOXD13 proteins [Williams et al., 2006], were transiently transfected with wildtype or mutant expression vector and transfection control plasmid $\beta$ actin- $\beta$ gal. Geldanamycin (kindly provided by W.B. Pratt and Yoshi Morishima) was used at nanomolar final concentrations and under the same protocol as previously described [Sittler et al., 2001]; final concentrations are shown in Figure 4. At $24 \mathrm{hr}$ post-transfection cells were collected by treatment with trypsin-EDTA and solubilized in detergent lysis $(100 \mathrm{mM}$ potassium phosphate [pH 7.8], 0.2\% Triton X-100, 1 mM DTT, and protease inhibitors [Roche Applied Sciences]) using the freeze thaw method. $\beta$-galactosidase enzyme activity was determined for each sample using the Promega $\beta$-galactosidase enzyme assay system. Sample volumes were normalized for $\beta$ galactosidase enzyme activity and separated by electrophoresis using 12\% SDS-polyacrylamide gels and subsequently electro-transferred to nitrocellulose. Blots were incubated with primary antibody at a 1:10,000 dilution of rabbit anti-HOXA13 [Post et al., 2000] and secondary antibodies at a 1:15,000 dilution of donkey anti-rabbit HRPconjugated secondary antibody (Amersham Biosciences, Piscataway, NJ). All antibodies were incubated with blots in PBST with 5\% Carnation nonfat dry milk. Protein expression was visualized using Supersignal chemiluminescent substrate (Pierce Biotechnology, Rockford, IL).

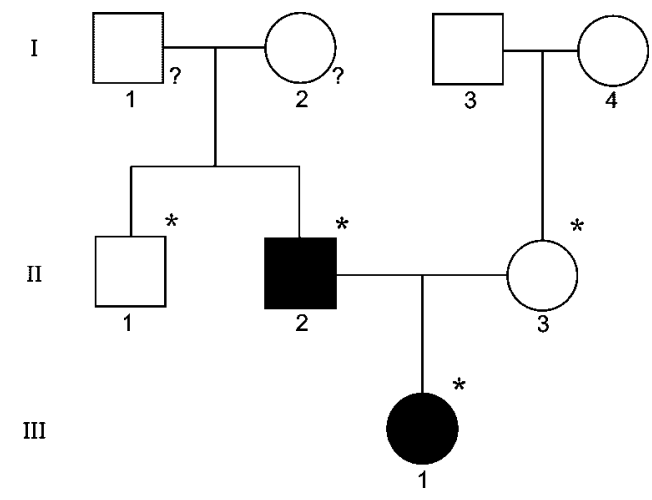

FIG. 1. Pedigree. Asterisks denote family members who were collected for mutational analysis of HOXA13. "?" refer to family members who were not examined and whose mutational status is unknown.

\section{RESULTS}

\section{Clinical Description and DNA Sequence Analysis}

The affected father (II-2, Fig. 1) presented with penoscrotal hypospadias, which was surgically repaired at 4 years of age, apparently hypoplastic thumbs and mild clinodactyly of the fifth fingers (Fig. 2). His feet were apparently small and flat. His great toes appeared short, as were the other toes. After having surgical correction of the medial deviation of his great toes, there remains slight lateral deviation.

The affected daughter (Fig. 2) presented with apparent shortening of her thumbs and fifth fingers with clinodactyly of the latter. Her great toes were also apparently short as were her feet. Like her father she had pes planus. In addition, she had left talipes equinovarus for which she underwent surgery at the age of 17 months. As an infant she always had a wet diaper, and she was subsequently found to have a neurogenic bladder and atonic detrusor. She also had a large, incompetent urethra. MRI studies of her lower spine revealed a fatty filum terminale, and during her second year of life she was diagnosed with a tethered cord, which was surgically corrected at 19 months. As a child, she has not achieved toilet training. By renal ultrasound there is no kidney structural abnormality. She has had a few urinary tract infections and, while these have not been serious problems, she has taken prophylactic antibiotics in the past. At 3.5 years she began intermittent catheterization, but her diapers remained wet. She does not have the urge to void, but she can feel catheter insertion. She had bilateral vesicoureteral reflux and underwent repair by transurethral polytetrafluoroethylene (Teflon) injection [Lopez et al., 1993]. She ultimately underwent surgical ureteral reimplantation as well as implantation of an artificial urinary sphincter to attempt to correct incontinence. She has a history of significant constipation and apparent lack of rectal sensation requiring parental rectal digital evacuation twice a day to prevent soiling. The anus is normally positioned and not stenotic. The labia and clitoris are normal. By direct exam, she had a longitudinal vaginal septum with narrow openings and two cervices, and she underwent vaginoplasty surgery. Her mother has a double uterus and delivered the proband by Cesarean. The mother did not have any history of miscarriages. The paternal grandparents were unavailable for exam. Additional family history was negative for others affected with HFGS.

Sequence analysis of the HOXA13 genes in the proband and her father revealed a heterozygous 42bp insertion in both (data not shown). This in-frame insertion introduces 14 additional alanines between the 17 th and 18 th residues of polyalanine tract III, which is usually 18 alanine residues in length. 

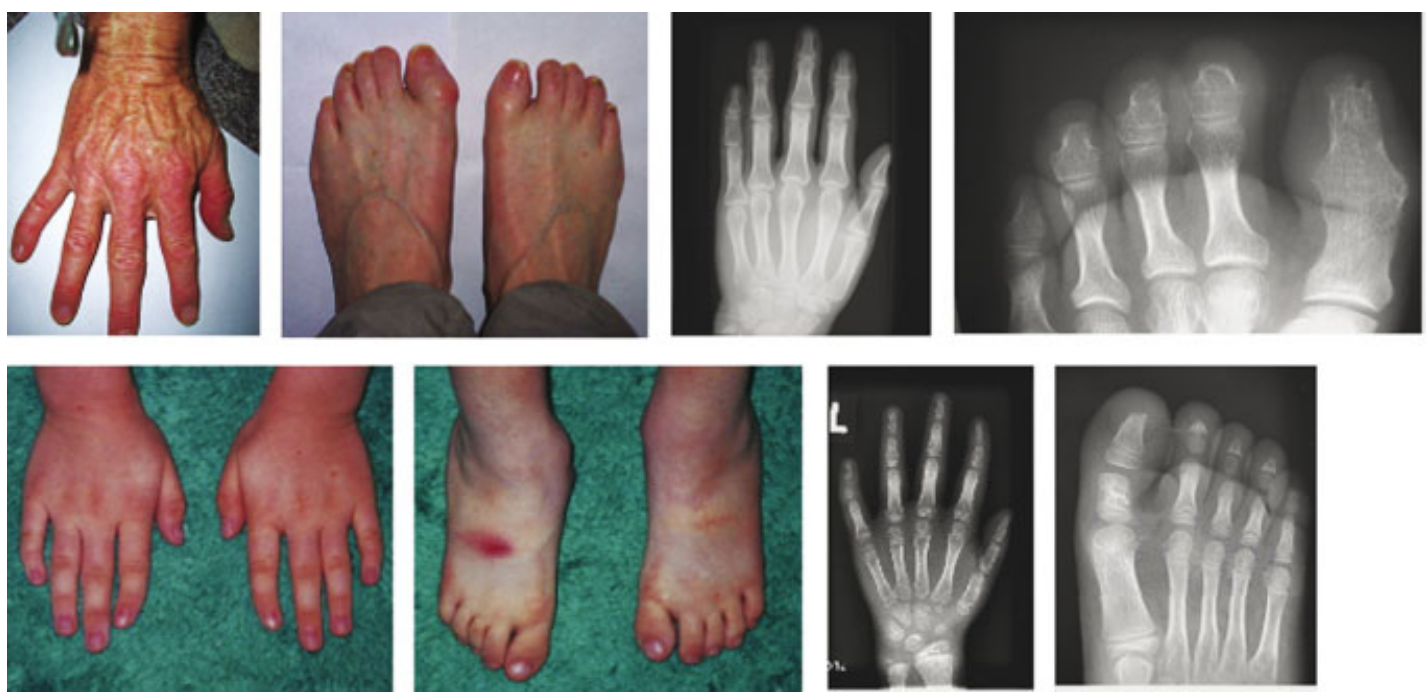

FIG. 2. Limb phenotypes of family members. Upper row, father; lower row, daughter showing hands and feet as well as radiographs, respectively. [Color figure can be viewed in the online issue, which is available at www.interscience.wiley.com.]

The expansion represents an exact duplication of the 4th to 17 th triplet of the wild-type sequence. The HOXA13 sequences of the mother and of the paternal uncle did not show any abnormalities.

\section{HOXA13 Mutant Polyalanine Expansion Proteins Aggregate in the Cytoplasm}

To study the mechanism whereby HOXA13 polyalanine expansion mutations result in disease, we transiently expressed wild-type HOXA13, +10 HOXA13, or +14 HOXA13 proteins in COS-7 cells and analyzed the distribution of the proteins by fluorescence microscopy. In these independent transfections, the wild-type protein was exclusively localized to the nucleus (Fig. 3A); however, the mutant proteins formed large aggregates in the cytoplasm in the majority of cells (Fig. 3B,C,F). The percent of cells with large cytoplasmic aggregates was greater for the +14 HOXA13 protein than the +10 HOXA13 protein. Cells expressing the mutant proteins displayed either nuclear or cytoplasmic staining, but not both. As a control, +14 HOXD13, but not wild-type HOXD13 (Fig. 3D) also showed cytoplasmic aggregates in the majority of cells as previously reported (Fig. 3E; [Albrecht et al., 2004]).

\section{Geldanamycin Reduces +10 HOXA13, +14 HOXA13, and +14 HOXD13 Steady-State Protein Abundance in Transfected Cells}

Geldanamycin is an Hsp90 inhibitor [reviewed in Pratt et al., 2004], and geldanamycin treatment of cells transfected with +14 HOXD13 results in reduction of aggregate formation and a commensurate increase in nuclear localization [Albrecht et al., 2004]. We confirmed cytoplasmic aggregation of +14 HOXD13 (Fig. 3E) and observed a slight geldana- mycin dose-dependent increase in nuclear localization of the mutant protein (data not shown).

To determine whether the +10 HOXA13 and +14 HOXA13 proteins responded the same way, we treated transfected cells similarly with increasing concentrations of geldanamycin. Forty hours after transfection in the presence of geldanamycin a substantial fraction of the cells were detached from the plate at nearly every geldanamycin concentration, which did not occur with wild-type HOXA13, HOXD13 or +14 HOXD13. By shortening the incubation time to $24 \mathrm{hr}$ we observed that the number of cells expressing the mutant protein decreased in a geldanamycin dose-dependent manner (data not shown) but without cell detachment. Moreover, there was no difference in the nuclear versus cytoplasmic localization of +10 or +14 HOXA13 proteins at any dose of geldanamycin.

To determine whether geldanamycin was reducing mutant HOXA13 expression by affecting transfection efficiency, we co-transfected cells with $\beta$ actin- $\beta$ gal and either wild-type HOXA13, +10 HOXA13, +14 HOXA13, wild-type HOXD13 or +14 HOXD13, and treated the transfected cells with increasing concentrations of geldanamycin. Cell lysates were collected at $24 \mathrm{hr}$ and assayed for $\beta$-galactosidase activity. Lysate volumes were normalized for $\beta$-galactosidase activity and analyzed by Western blot analysis with anti-HOXA13 or anti-HOXD13 antibody (Fig. 4). Steady-state expression of wild-type HOXA13 or HOXD13 was not affected by geldanamycin treatment; however, +10 HOXA13, +14 HOXA13 and +14 HOXD13 demonstrated a dose-dependent decrease in steady-state mutant protein abundance. Steady-state abundance was reduced with the following gradient: +14 HOXD13 $\leq+10$ HOXA13 < +14 HOXA13. Thus, reduction in polyalanine expanded HOX protein steady-state concentration 

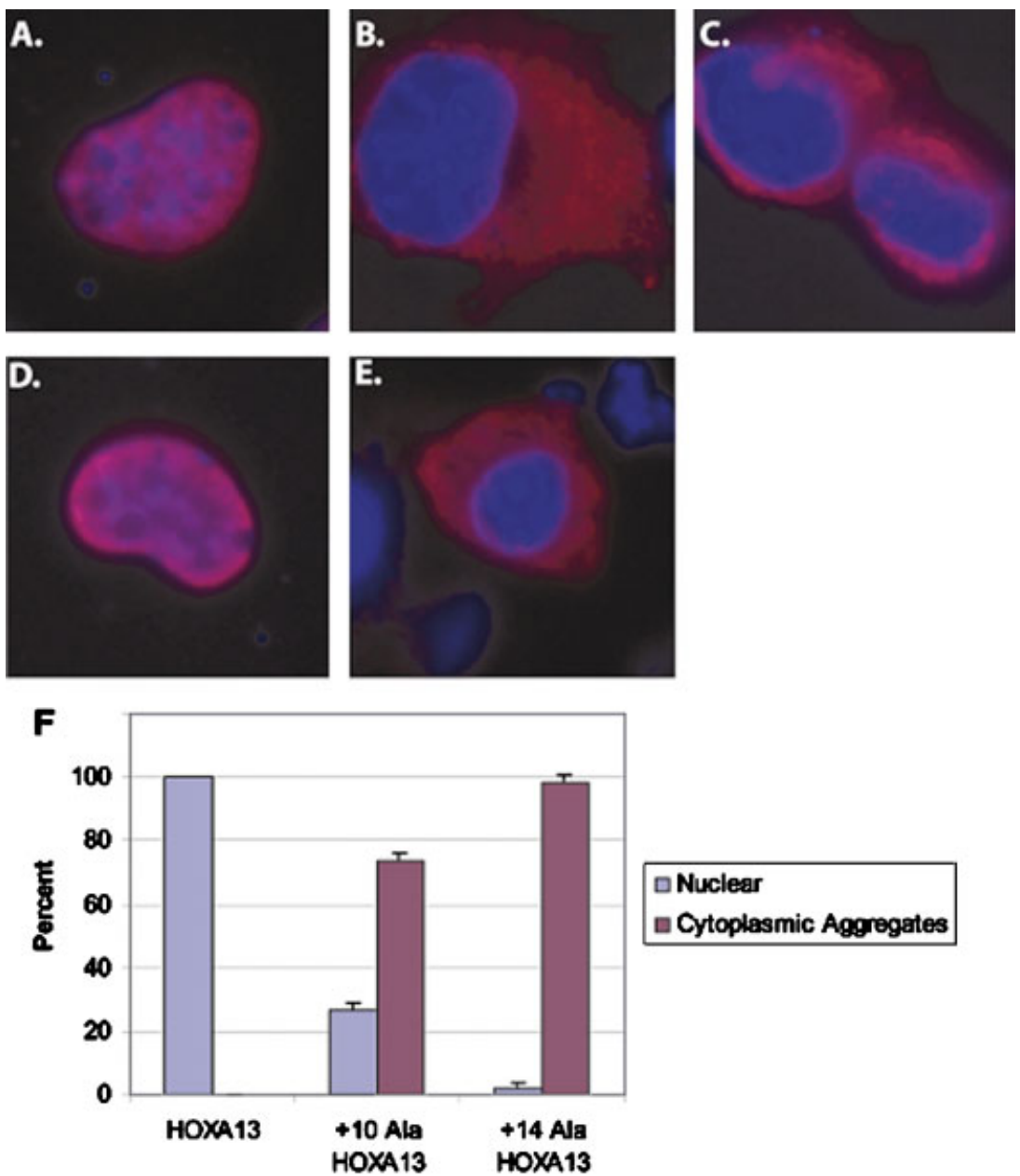

FIG. 3. HOXA13 and HOXD13 alanine expansion mutants form cytoplasmic aggregates. Nuclei are stained with DAPI (blue); proteins, red. Wild-type HOXA13 (A) and HOXD13 (D) are localized to the nucleus. $+10 \operatorname{HOXA13}(\mathbf{B}),+14$ HOXA13 $(\mathbf{C})$, and +14 HOXD13 (E) result in cytoplasmic aggregates. F: Subcellular distribution of wild-type, +10 , and +14 HOXA13. Increasing alanine tract length results in a greater percentage of cells with cytoplasmic protein localization. [Color figure can be viewed in the online issue, which is available at www.interscience.wiley.com.]

in cells treated with geldanamycin is not secondary to reduction in transfection efficiency, and +14 HOXA13 exhibited the least abundance.

\section{Wild-Type HOXA13 or HOXD13 Mislocalize Upon Co-Expression of Mutant HOXA13 Proteins}

In co-expression experiments, wild-type HOXD13 was shown to co-localize with polyalanine expanded HOXD13 in cellular aggregates [Albrecht et al., 2004], which was hypothesized to be a potential explanation for the dominant nature of progressively larger HOXD13 polyalanine expansions. They also showed that wild-type HOXD11 and HOXD12 are not trapped in polyalanine expanded HOXD13 aggregates but localize properly to the nucleus. WT HOXA13 was similarly reported to aggregate in the cytoplasm with polyalanine expanded HOXA13 but apparently WT HOXA13 did not aggregate in the cytoplasm with the +14 HOXD13 [Albrecht et al., 2004]. We sought to explore cytoplasmic coaggregation in relation to HOXA13.

To investigate the effect of +10 HOXA13 or +14 HOXA13 polyalanine expansion proteins on wildtype HOXA13 protein localization, we coexpressed FLAG-tagged wild-type HOXA13 and either +10 HOXA13 or +14 HOXA13 in COS-7 cells. Upon coexpression of +10 HOXA13, 32\% of cells mislocalized the wild-type HOXA13 protein to cytoplasmic aggregates, while coexpression of +14 HOXA13 resulted in 69\% of cells mislocalizing the wild-type protein to cytoplasmic aggregates (Fig. 5). In contrast, cytoplasmic aggregation was observed in $73 \%$ of cells expressing + 10 HOXA13 alone and 98\% expressing + 14 HOXA13 alone. Although this difference may be explained by the rates of production of the mutant and wild-type proteins in these cells, wild-type HOXA13 was sequestered in the cytoplasmic aggregates as expected. 
3166

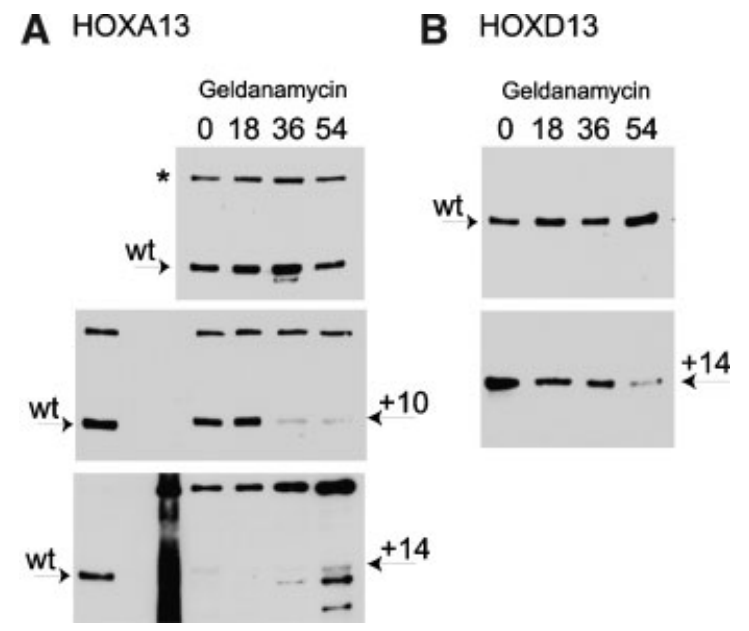

FIG. 4. Geldanamycin treatment results in decreased protein expression of HOXA13 with alanine expansions. COS- 7 cells transfected with $\beta$-actin- $\beta$ galactosidase control plasmid and either (A) wild-type HOXA13, +10 HOXA13, or +14 HOXA13 or $(\mathbf{B})$ wild-type HOXD13 or +14 HOXD13 were treated with increasing concentrations, denoted above lanes, of geldanamycin (nM). Cell lysate volumes were normalized for transfection efficiency by $\beta$-galactosidase activity and used in Western blot analysis. Upper panels show the effect of geldanamycin on wild-type steady-state abundance. Wild-type HOXA13 and HOXD13 transfections were performed in parallel in middle and lower panels and lysate protein was included on the same gel to demonstrate the higher molecular weight of the mutant polyalanine proteins. At the higher geldanamycin concentrations, full length +14 HOXA13 protein abundance is very low, but shorter molecular weight immunoreactive products, presumably made from alternative in-frame initiator methionines, which are produced in very low quantities in wild-type transfections, become more apparent as a result of loading normalization. Asterisk in HOXA13 experiments denotes a crossreacting protein band that is not HOXA13.

To explore whether polyalanine expanded HOXA 13 affects the localization of wild-type HOXD13 protein we coexpressed Flag-tagged wild-type HOXD13 with either +10 HOXA13 (Fig. 6A), +14 HOXA13 (Fig. 6B), or wild-type HOXA13 (Fig. 6C). We found that wild-type HOXD13 was co-localized with polyalanine expanded HOXA13 in cytoplasmic aggregates in $26 \%$ of cells for +10 HOXA13 and $75 \%$ of cells with +14 HOXA13, but not with wild-type HOXA13. The results show that HOXA13 polyala- nine expansion proteins are capable of sequestering, at least in this assay, wild-type HOXA13 as well as wild-type HOXD13 proteins in cytoplasmic aggregates. These data are not compatible with the hypothesis that the dominant nature of HOXD13 polyalanine expansions occurs because of sequestration of wild-type HOXD13 and/or HOXA13. These results also demonstrate a length dependency for HOXA13 polyalanine tract expansion proteins to sequester wild-type proteins to cytoplasmic aggregates.

\section{DISCUSSION}

The mutation presented here represents the largest polyalanine expansion so far observed in HOXA13 [Innis et al., 2004] or for any other protein [Brown and Brown, 2004]. Manifestations found in the proband not reported in other HFGS patients include tethered cord, unilateral talipes equinovarus, and constipation requiring manual evacuation. Given previous clinical reports and the expression pattern of HOXA13 in the urogenital sinus and the Mullerian ducts it is not surprising to find neurogenic bladder, atonic detrusor, bilateral ureteral reflux, patulous urethra as well as double uterus and vagina with small hymenal openings [Innis, 2004]. Other than penoscrotal hypospadias the absence of severe urogenital manifestations in the father is consistent with the variability of the genitourinary problems in patients with HFGS [Innis, 2004]. Without additional information about paternal grandparental mutational status, we must hold out somatic mosaicism in the proband's father as one possibility for the variation as well. While tethered cord has not been reported in other HFGS patients, it would be important to consider this in HFGS patients. Similarly, a history of constipation in patients with HFGS should be sought and, if present, appropriately managed.

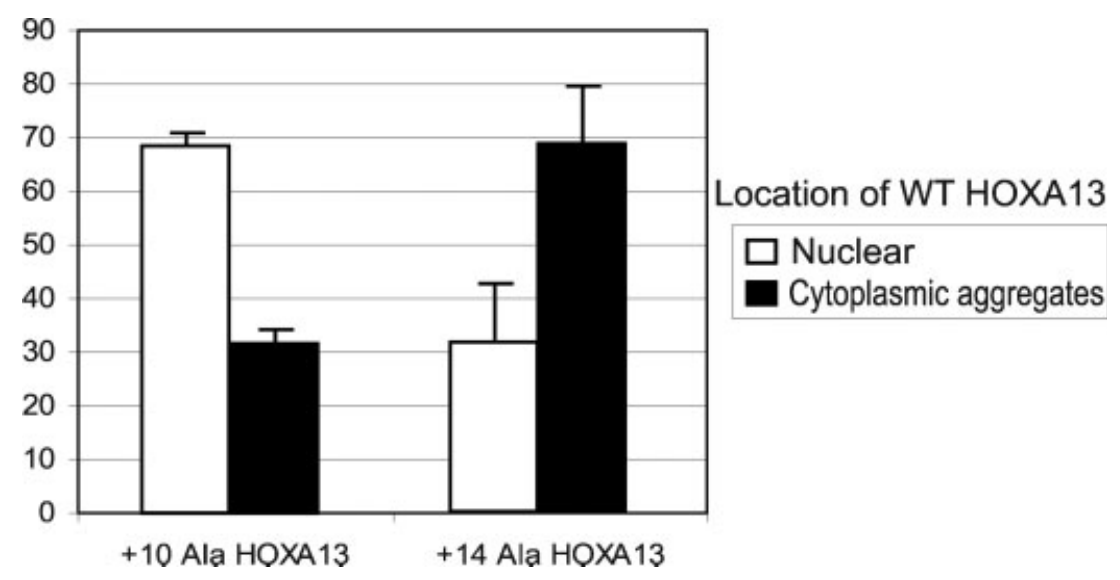

FIG. 5. Co-expression of wild-type HOXA13 with HOXA13 polyalanine expansion mutant proteins. Coexpression of FLAG-tagged wild-type HOXA13 with either +10 HOXA13 or +14 HOXA13 results in localization of wild-type protein to cytoplasmic aggregates. Y-axis = percentage of cells. 
A
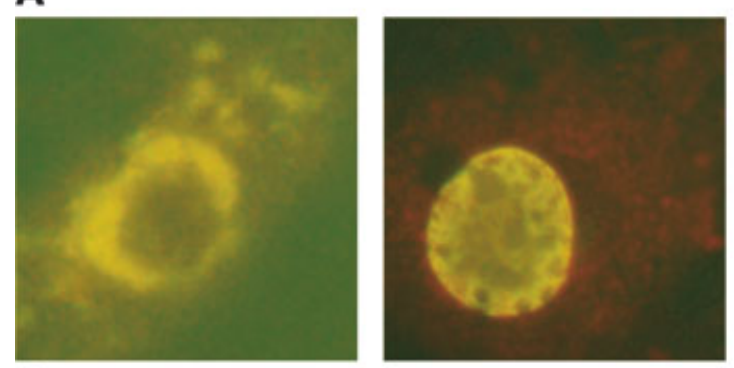

B
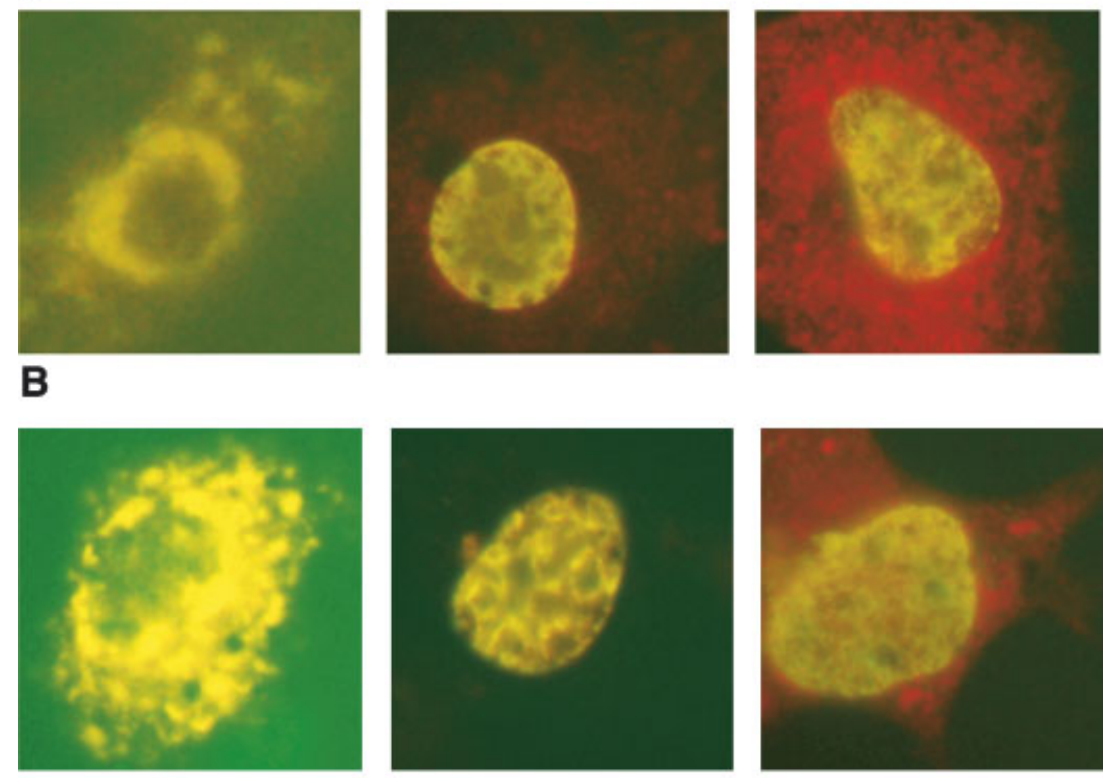

C

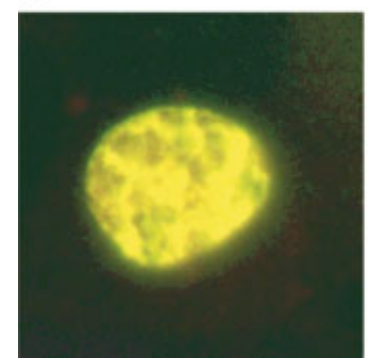

FIG. 6. Wild-type HOXD13 colocalizes with HOXA13 alanine expansion mutant proteins. All panels are representative examples of coexpression patterns in cells. All are merged images of HOXD13-FLAG visualized with a FITC-conjugated antibody and mutant or wild-type HOXA13 visualized with rhodamine-conjugated antibody. A: FLAG-HOXD13 transiently coexpressed with +10 HOXA13. Left panel, HOXD13 in cytoplasmic aggregates with mutant HOXA13 was observed in $25 \%$ of cells; Middle panel, HOXD13 and mutant HOXA13 co-localized in the nucleus ( $73 \%$ of cells); Right panel, HOXD13 localized in the nucleus and mutant HOXA13 in cytoplasmic aggregates ( $1-2 \%$ of cells). B: FLAG-HOXD13 transiently coexpressed with +14 HOXA13. Co-localization in the cytoplasm (left panel, $75 \%$ of cells) or together in the nucleus in some cells (middle panel, 24\%) or rarely (right panel, $\leq 1 \%$ ) HOXD13 in the nucleus and mutant HOXA13 in the cytoplasm. C: Wild-type HOXD13-FLAG and wild-type HOXA13 proteins colocalize to the nucleus in 100\% of cells. A: +10Ala HOXA13 coexpression with wild-type HOXD13. B: +14Ala HOXA13 coexpression with wild-type HOXD13. C: Wild-type HOXA13 coexpression with wild-type HOXD13. [Color figure can be viewed in the online issue, which is available at www.interscience.wiley.com.]

We have replicated the findings of Albrecht et al. [2004] in demonstrating the accumulation of cytoplasmic aggregates of polyalanine expanded HOXA13 and HOXD13 proteins. However, we have also shown a polyalanine length dependence on the frequency of cytoplasmic aggregation not previously shown for HOXA13.

In addition, the steady-state abundance of +10 HOXA13 (exhibiting a total tract length of 28) appeared in our hands to be reduced more in the presence of geldanamycin than +14 HOXD13 (total tract length 29). Thus, total alanine tract length is not the only determinant of protein fate. Also, we observed no difficulty with cell attachment after transfection of only +10 or +14 HOXA13 alone, however, the addition of geldanamycin to the experiments resulted in extensive cell detachment. This was not observed with +14 HOXD13 in the presence of geldanamycin. Inhibition of hsp90 by geldanamycin can result in a wide array of effects but increases degradation of misfolded proteins [Schneider et al., 1996; Pratt et al., 2004; Thomas et al., 2006]. It is attractive to speculate that HOXA13 polyalanine expanded proteins are more unstable than comparable length expansions in HOXD13, that geldanamycin treatment shifts polyalanine expanded HOXA13 proteins more efficiently to the degradation pathway or that misfolding is more likely to be terminal for HOXA13, yet potentially correctable with HOXD13 expansions. Whether or not this reflects an underlying variation in the in vivo disposition of mutant HOXA13 and HOXD13 proteins remains to be determined.

Finally, mutant HOXA13 proteins of +10 or +14 alanines sequester wild-type HOXA13, as expected, but in contrast to the results of Albrecht et al. [2004], 
also sequester wild-type HOXD13. Therefore, differential sequestration of wild-type HOX protein paralogs in cytoplasmic aggregates does not explain the difference between the apparent loss of function that occurs with polyalanine expansions of HOXA13 versus the dominant-negative nature attributed to HOXD13 polyalanine expansions of increasing size. Rather, the difference may be in coaggregation of other transcription cofactors or in cellular mechanisms that affect the nuclear function or localization, refolding or degradation rates of mutant proteins.

\section{ACKNOWLEDGMENTS}

We wish to thank the family for their patience and help during participation in this study. We thank Stefan Mundlos, Berlin, Germany for generously providing us with the mouse +14 HOXD13 clone. Thanks to W.B. Pratt and Y. Morishima, Department of Pharmacology, University of Michigan, for providing geldanamycin and for helpful discussions. Thanks to A. Lieberman for helpful discussion and critical review of the manuscript. Thanks to $\mathrm{R}$. Brunner, Kantonsspital Bruderholz, Basel, Switzerland for providing us with radiographs of the family members. Thanks to U. Jacobs, Children's Hospital, University of Erlangen-Nuremberg, Germany for technical assistance. B.U. was supported in part by the "Arbeitsgemeinschaft Pädiatrische Nephrologie". Colleen McCabe was supported by the University of Michigan Office of the Vice President for Research and the Department of Human Genetics.

\section{REFERENCES}

Albrecht A, Mundlos S. 2005. The other trinucleotide repeat: Polyalanine expansion. Curr Opin Genet Dev 15:285-293.

Albrecht AN, Kornak U, Boddrich A, Suring K, Robinson PN, Stiege AC, Lurz R, Stricker S, Wanker EE, Mundlos S. 2004. A molecular pathogenesis for transcription factor associated poly-alanine tract expansions. Hum Mol Genet 13:2351-2359.

Brown LY, Brown SA. 2004. Alanine tracts: The expanding story of human illness and trinucleotide repeats. Trends Genet 20: $51-58$.

Innis JW. 2004. HOXA13 and the hand-foot-genital and Guttmacher syndromes. In: Epstein C, Erickson R, Wynshaw-Boris A, editors. Inborn errors of development: The molecular basis of clinical disorders of morphogenesis. Oxford, UK: Oxford University Press. p 529-539.

Innis JW, Mortlock D, Chen Z, Ludwig M, Williams ME, Williams TM, Doyle CD, Shao Z, Glynn M, Mikulic D, Lehmann K, Mundlos S, Utsch B. 2004. Polyalanine expansion in HOXA13: Three new affected families and the molecular consequences in a mouse model. Hum Mol Genet 13:2841-2851.

Kjaer KW, Hansen L, Eiberg H, Utkus A, Skovgaard LT, Leicht P, Opitz JM, Tommerup N. 2005. A 72-year-old Danish puzzle resolved-Comparative analysis of phenotypes in families with different-sized HOXD13 polyalanine expansions. Am J Med Genet Part A 138A:328-339.

Kjaer KW, Hedeboe J, Bugge M, Hansen C, Friis-Henriksen K, Baeksted Vestergaard M, Tommerup N, Opitz JM. 2002. HOXD13 polyalanine tract expansion in classical synpolydactyly type Vordingborg. Am J Med Genet 110:116-121.

Lavoie H, Debeane F, Trinh Q-D, Turcotte J-F, Corbeil-Girard L-P, Dicaire M-J, Saint-Denis A, Page M, Rouleau GA, Brais B. 2003. Polymorphism, shared functions and convergent evolution of genes with sequences coding for polyalanine domains. Hum Mol Genet 12:2967-2979.

Lopez AE, Padron OF, Patsias G, Politano VA. 1993. Transurethral polytetrafluoroethylene injection in female patients with urinary incontinence. J Urol 150:856-858.

McCabe C, Innis JW. 2005. A genomic approach to identification and characterization of HOXA13 functional binding elements. Nucleic Acids Res 33:6782-6794.

Mortlock DP, Innis JW. 1997. Mutation of HOXA13 in hand-footgenital syndrome. Nat Genet 15:179-180.

Post LC, Margulies EH, Kuo A, Innis JW. 2000. Severe limb defects in Hypodactyly mice result from expression of a novel, mutant HOXA13 protein. Dev Biol 217:290-300.

Pratt WB, Galigniana MD, Harrell JM, DeFranco DB. 2004. Role of hsp90 and the hsp90-binding immunophilins in signaling protein movement. Cell Signal 16:857-872.

Schneider C, Sepp-Lorenzino L, Nimmesgern E, Qoerfelli O, Danishefsky S, Rosen N, Hartl FU. 1996. Pharmacologic shifting of a balance between protein refolding and degradation mediated by Hsp90. Proc Natl Acad Sci USA 93:1453614541.

Sittler A, Lurz R, Lueder G, Priller J, Lehrach H, Hayer-Hartl MK, Hartl FU, Wanker EE. 2001. Geldanamycin activates a heat shock response and inhibits huntingtin aggregation in a cell culture model of Huntington's disease. Hum Mol Genet 10: $1307-1315$.

Thomas M, Harrell JM, Morishima Y, Peng H-M, Pratt WB, Lieberman AP. 2006. Pharmacologic and genetic inhibition of hsp90-dependent trafficking reduces aggregation and promotes degradation of the expanded glutamine androgen receptor without stress protein induction. Hum Mol Genet 15:1876-1883.

Williams ME, Lehoczky JA, Innis JW. 2006. A group 13 homeodomain is neither necessary nor sufficient for posterior prevalence in the mouse limb. Dev Biol 297:493-507. 